\title{
Electrophysiologic Effects of Sotalol and Amiodarone in Patients with Sustained Monomorphic Ventricular Tachycardia
}

\author{
K. Ching Man, DO, Brian D. Williamson, MD, Mark Niebauer, MD, Emile Daoud, MD, \\ Omar Bakr, MD, S. Adam Strickberger, MD, John D. Hummel, MD, \\ William Kou, MD, and Fred Morady, MD
}

\begin{abstract}
No prospective studies have compared sotalol and amiodarone during electropharmacologic testing. The purpose of this prospective, randomized study was to compare the electrophysiologic effects of sotalol and amiodarone in patients with coronary artery disease and sustained monomorphic ventricular tachycardia (VT). Patients with coronary artery disease and sus. tained monomorphic VT inducible by programmed stimulation were randomly assigned to receive either sotalol ( $n=17$ ) or amiodarone $(n=17)$. The sotalol dose was titrated to $240 \mathrm{mg}$ twice daily over 7 days. Amiodarone dosing consisted of $600 \mathrm{mg} 3$ times daily for 10 days. An electrophysiologic test was performed in the baseline state and at the end of the loading regimen. An adequate response was defined as the inability to induce $V T$ or the ability to induce only relatively slow hemodynamically stable VT. During the follow-up electrophysiologic test, $24 \%$ of patients taking sotalol and $41 \%$ of those taking amiodarone had an adequate response to therapy $(p=0.30)$. Amiodarone lengthened the mean VT cycle length to a greater degree than sotalol ( $28 \%$ vs $12 \%$, p <0.01). There were no significant differences in the effects of sotalol and amiodarone on the ventricular effective refractory period. In patients with coronary artery disease, amiodarone and sotalol are similar in efficacy in the treatment of VT as assessed by electropharmacologic testing. The effects of the 2 drugs on ventricular refractoriness are similar, but amiodarone slows VT to a greater extent than sotalol.
\end{abstract}

(Am J Cardiol 1994;74:1119-1123)

From the Department of Internal Medicine, Division of Cardiology, The University of Michigan Medical Center, Ann Arbor, Michigan. Manuscript received May 25, 1994; revised manuscript received and accepted July 19,1994

Address for reprints: Fred Morady, MD, University of Michigan Medical Center, 1500 East Medical Center Drive, B1F245, Ann Arbor, Michigan 48109-0022.
7 The results of a large scale, prospective study suggested that sotalol may be more efficacious than several other antiarrhythmic agents in controlling ventricular tachycardia (VT). ${ }^{1}$ However, no prior studies have prospectively compared sotalol and amiodarone in the electrophysiology laboratory. The purpose of this study was to compare prospectively, in a randomized fashion, the electrophysiologic effects of sotalol and amiodarone in a homogenous group of patients with coronary artery disease and inducible sustained monomorphic VT undergoing electropharmacologic testing.

\section{METHODS}

Patients: The subjects in this study were 40 consecutive patients ( $32 \mathrm{men}$ and 8 women, mean age \pm SD $64 \pm 9$ years) with coronary artery disease and sustained monomorphic VT inducible by programmed ventricular stimulation. Mean left ventricular ejection fraction as measured by echocardiography or radionuclide ventriculography was $0.34 \pm 0.09$. Twenty-three patients presented with sustained VT, 13 patients presented with symptomatic, nonsustained VT, 2 patients presented with aborted sudden death, and 2 patients presented with syncope. Exclusion criteria included: (1) left ventricular ejection fraction $<0.20$; (2) congestive heart failure unresponsive to conventional therapy; (3) pulmonary disease contraindicating the use of amiodarone; (4) liver disease or renal failure; (5) previous intolerance to $\beta$ blockers; and (6) clinically significant sinus node dysfunction or atrioventricular block. There were no significant differences in the clinical characteristics of the patients treated with sotalol and amiodarone.

Electrophysiologic testing: Informed, written consent was obtained from each patient under a protocol approved by the Human Research Committee at the University of Michigan. Each patient underwent a baseline electrophysiologic test after discontinuation of all antiarrhythmic agents for $\geq 5$ half-lives. Quadripolar electrode catheters were inserted into a femoral vein and positioned in the high right atrium, His bundle position, and right ventricular apex. Leads $\mathrm{V}_{1}, \mathrm{I}, \mathrm{II}$, and III, and the intracardiac electrograms were displayed on an oscilloscope and recorded at a paper speed of 25 to $100 \mathrm{~mm} / \mathrm{s}$ on a Mingograph 7 recorder (Siemens-Elema, Inc., Solna, Sweden). Pacing was performed at twice diastolic threshold with a pulse duration of $2 \mathrm{~ms}$ using a programmable stimulator (Bloom Associates, Ltd., Reading, Pennsylvania). In all patients, the ventricular capture threshold was $<1 \mathrm{~mA}$.

The $\mathrm{AH}$ and $\mathrm{HV}$ intervals were measured during atrial pacing at a cycle length of $600 \mathrm{~ms}$. The atrioventric- 
ular block cycle length was determined by incremental atrial pacing in steps of $10 \mathrm{~ms}$. The ventricular effective refractory period was measured at the right ventricular apex using a basic drive cycle length of $600 \mathrm{~ms}$. The right atrium and ventricle were paced simultaneously using an 8-beat drive train and a 4-second intertrain pause. A 1-minute conditioning train was used before the refractory periods were measured. The effective refractory period was measured by scanning diastole after each 8-beat drive train wilh a single ventricular extrastimulus. The coupling interval of the extrastimulus was shortened in $10 \mathrm{~ms}$ steps until there was loss of capture, and then lengthened in $2 \mathrm{~ms}$ steps until ventricular capture occurred. The effective refractory period was defined as the longest coupling interval that did not result in ventricular capture. A prior study ${ }^{2}$ demonstrated that this technique for determination of the ventricular effective refractory period results in measurements that are reproducible to within $4 \mathrm{~ms}$.

The stimulation protocol used to induce VT has been described in detail previously. ${ }^{3}$ Programmed stimulation was initially performed at the right ventricular apex. One to 3 extrastimuli were delivered after an 8-beat drive train using a basic drive cycle length of $350 \mathrm{~ms}$, then 400 and $600 \mathrm{~ms}$. If sustained VT was not induced, the stimulation protocol was repeated at the right ventricular septum or outflow tract. Whenever sustained VT was induced, a 12-lead electrocardiogram was recorded. In patients in whom multiple types of VT were induced, the VT that had the shortest cycle length was used for analysis.

Sustained, monomorphic VT was defined as VT of uniform morphology lasting $>30$ seconds or requiring termination because of cardiovascular collapse. Hemodynamically stable VT was defined as VT associated with a systolic pressure $>90 \mathrm{~mm} \mathrm{Hg}$ and no symptoms of cerebral hypoperfusion. An adequate drug response was defined as the ability to induce only VT that had a cycle length of $\geq 350 \mathrm{~ms}$ and was hemodynamically stable, ${ }^{4-6}$ or as the inability to induce VT. ${ }^{7-9}$ The end points of the stimulation protocol were the induction of hemodynamically unstable sustained monomorphic VT, repeated induction of only hemodynamically stable sustained monomorphic VT, or completion of the pacing protocol at 2 right ventricular sites.

Drug therapy and electropharmacologic testing: Patients were randomly assigned to receive either sotalol or amiodarone. Both drugs were administered orally. Twenty-one patients assigned to receive sotalol were treated with $80 \mathrm{mg}$ every 12 hours for 4 doses, $160 \mathrm{mg}$ every 12 hours for 4 doses, then with $240 \mathrm{mg}$ every 12 hours for 5 doses, if tolerated. Nineteen patients assigned to receive amiodarone were treated with $600 \mathrm{mg}$ every 8 hours for 10 days. None of the patients were treated with any other antiarrhythmic drugs or $\beta$ blockers.

All patients underwent continuous, telemetric electrocardiographic monitoring and were questioned daily regarding the adverse effects of sotalol or amiodarone. Sotalol was discontinued in 1 patient due to heart failure, in 2 patients due to symptomatic bradycardia, and in 1 patient because of torsades de pointes. The remaining 17 patients were able to tolerate a dosage of $240 \mathrm{mg}$ of sotalol twice daily and underwent a follow-up elec- trophysiologic test after 7 days of therapy. Amiodarone was discontinued in 1 patient due to proarrhythmia and in 1 patient who developed an acute myocardial infarction. One patient experienced nausea on day 3 of amiodarone therapy and the dose was reduced to $400 \mathrm{mg} 3$ times daily. A follow-up electrophysiologic test was performed after 10 days of amiodarone therapy in 17 patients.

Statistical analysis: Values are expressed as mean \pm SD. Comparisons of continuous variables were performed either using Student's paired $t$ test or by analysis of variance. Categorical variables were compared by chi-square analysis. A p value $<0.05$ was considered significant.

\section{RESULTS}

Inducibility of ventricular tachycardla: All 34 patients who successfully completed the loading regimens of sotalol and amiodarone had inducible, hemodynamically unstable sustained monomorphic VT in the baseline state. The mean VT cycle length was $268 \pm 32 \mathrm{~ms}$. VT had a right bundle branch block configuration in 22 patients and a left bundle branch block configuration in 12 paticnts. VT was induced by triple extrastimuli in 23 patients and by double extrastimuli in 11 patients. There were no significant baseline differences in VT characteristics between those taking sotalol and those taking amiodarone at the time of randomization.

Sotalol was efficacious in 4 of 17 patients (24\%), with efficacy manifest as complete suppression of inducible VT in each of the 4 patients. VT was induced by triple extrastimuli in 8 patients taking sotalol and by double extrastimuli in 5 taking sotalol. Amiodarone was efficacious in 7 of 17 patients (41\%). Among the 17 patients treated with amiodarone, VT was not inducible in $3 \mathrm{pa}-$ tients $(18 \%)$ and inducible but hemodynamically stable in 4 patients $(24 \%)$. VT was induced by triple extrastimuli in 10 patients taking amiodarone and by double extrastimuli in 4 taking amiodarone. The overall efficacy of sotalol and amiodarone did not differ significantly $(\mathrm{p}=0.30)$.

Ventricular tachycardia cycle length: The mean VT cycle length was prolonged significantly both by sotalol ( $p<0.01$ ) and by amiodarone $(p<0.001)$ compared with baseline. The magnitude of prolongation in the mean VT cycle length was $41 \mathrm{~ms}$ greater with amiodarone than with sotalol $(\mathrm{p}<0.01)$. The mean VT cycle length in the 13 patients who still had inducible VT after treatment with sotalol increased by $12 \%$, from $274 \pm 42$ to $306 \pm 33 \mathrm{~ms}(\mathrm{p}<0.01)$, and none of the VTs were hemodynamically stable. In contrast, among the 14 patients taking amiodarone who still had inducible VT, the mean VT cycle length increased by $28 \%$, from 269 \pm 26 to $344 \pm 44 \mathrm{~ms}(\mathrm{p}<0.001)$. Among the 4 patients who had inducible but hemodynamically stable V'T after treatment with amiodarone, the mean VT cycle length increased by $47 \%$, from $265 \pm 13$ to $390 \pm 50 \mathrm{~ms}$ (p $<0.001$ ).

Ventricular effective refractory periods: Sotalol and amiodarone both significantly lengthened the ventricular effective refractory period (Figure 1). The mean ventricular effective refractory period in the sotalol group 
increased by $19 \%$, from $250 \pm 18$ to $297 \pm 25 \mathrm{~ms}$ (p $<0.001$ ). Similarly, the ventricular effective refractory period in the amiodarone group increased by $15 \%$, from $262 \pm 26$ to $301 \pm 33 \mathrm{~ms}(\mathrm{p}<0.001)$. There was no significant difference in the magnitude of the effects of sotalol and amiodarone on the ventricular effective refractory period.

In patients in whom there was an adequate drug response, the mean ventricular effective refractory period in the sotalol group increased by $25 \%$, from $253 \pm$ 26 to $317 \pm 28 \mathrm{~ms}(\mathrm{p}<0.05)$ and the ventricular effective refractory period in the amiodarone group increased by $22 \%$, from $254 \pm 22$ to $310 \pm 27 \mathrm{~ms}(\mathrm{p}<0.05)$. There was no significant difference in the effects of amiodarone and sotalol on the ventricular effective refractory period between the patients who responded to drug therapy and those who did not.

Other electrophysiologic effects (Figure 1): Sotalol lengthened the mean sinus cycle length by $42 \%$, from $786 \pm 104$ to $1,113 \pm 123 \mathrm{~ms}(\mathrm{p}<0.001$ ), and amiodarone increased the mean sinus cycle length by $19 \%$, from 821 \pm 188 to $975 \pm 167 \mathrm{~ms}(\mathrm{p}<0.01)$. The effect of sotalol on the sinus cycle length was greater than that of amiodarone $(\mathrm{p}<0.05)$.

Sotalol lengthened the AH interval by $22 \%$, from 108 \pm 44 to $132 \pm .36 \mathrm{~ms}(\mathrm{p}<0.05)$, and amiodarone increased the mean AH interval by $35 \%$, from $128 \pm 63$ to $173 \pm$ $78 \mathrm{~ms}(\mathrm{p}<0.01)$ during atrial pacing at a cycle length of $600 \mathrm{~ms}$. The effects of the 2 drugs on $\mathrm{AH}$ interval did not differ significantly.

Sotalol lengthened the mean atrioventricular block cycle length by $28 \%$, from $362 \pm 51$ to $462 \pm 66 \mathrm{~ms}$ (p $<0.001$ ) and amiodarone increased the mean atrioventricular block cycle length by $23 \%$, from $454 \pm 132$ to $557 \pm 121 \mathrm{~ms}(\mathrm{p}<0.001)$. The effects of the 2 drugs on the atrioventricular block cycle length did not differ significantly.

\section{DISCUSSION}

Major findings: The major finding of this randomized study is that sotalol and amiodarone were similar in efficacy for the control of VT as assessed by electrophysiologic testing in patients with coronary artery disease. Although the 2 drugs were similar in their ability to suppress the induction of VT, amiodarone was more effective than sotalol in slowing the rate of VT in patients in whom VT remained inducible despite drug therapy.

Effects of sotalol and amiodarone on ventricular tachycardia cycle length: The sotalol group had a $12 \%$ increase in the mean VT cycle length compared with $28 \%$ in the amiodarone group. This greater increase in cycle length in the amiodarone group resulted in VT becoming hemodynamically stable in approximately $25 \%$ of patients. In this subgroup of amiodarone patients, the mean VT cycle length increased by $43 \%$ compared with baseline. In contrast, induced VT did not become hemodynamically stable in any of the patients treated with sotalol who still had inducible VT.

The results of this study are in accord with the results of Nademanee et al, ${ }^{10}$ who found that sotalol increased the mean VT cycle length by $20 \%$. In addition, in a previous study, amiodarone was reported to increase the mean VT cycle length by $33 \%,{ }^{6}$ also in concert with the results of the present study. These findings suggest that amiodarone may slow conduction in the myocardium to a greater extent than sotalol.

Ventricular effective refractory periods: In the present study, sotalol and amiodarone were found to lengthen the ventricular effective refractory period to a similar degree, and the extent of prolongation in the ventricular effective refractory period did not correlate with the ability of the drug to suppress the induction of VT. This finding is consistent with the results of Kuchar et al, ${ }^{11}$ who reported that changes in the ventricular effective refractory period were not an indicator of the response to sotalol in patients with VT. Additionally, in another study, the effects of amiodarone on the ventricular effective refractory period also were found not to correlate with its effects on the induction of VT. ${ }^{12}$

Effects on sinus cycle length: The sinus cycle length was prolonged by a mean of almost $50 \%$ with sotalol compared with only approximately $20 \%$ with amiodarone. The greater depressant effect of sotalol on sinus node function was manifest by the occurrence of symptomatic bradycardia requiring discontinuation of therapy in 2 patients. This was the case despite the exclusion from this study of patients with clinical evidence of sinus node function. The greater degree of slowing of the sinus rate with sotalol is likely to be a reflection of its $\beta$ adrenergic-blocking activity.

Adverse drug effects: A significant degree of $\beta$ adrenergic blockade by sotalol was manifest not only by the occurrence of symptomatic bradycardia, but also by the occurrence of congestive heart failure in a patient being treated with sotalol. Although amiodarone also has

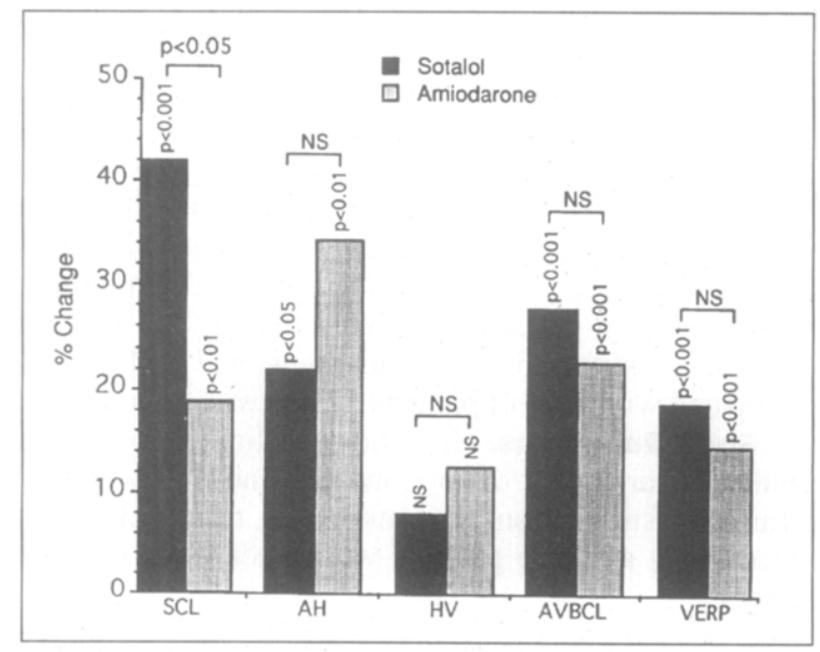

FIGURE 1. The electrophysiologic effects of sotalol (17 patients) and amiodarone (17 patients). Vertical axis indicates the percent change compared with baseline for each of the variables shown. The values above each bar refer to the significance of the drug effect, and the values spanning adjacent bars refer to the comparison between sotalol and amlodarone. AH = Interval measured during atrial pacing at a cycle length of $600 \mathrm{~ms}$; AVBCL = atrioventricular nodal block cycle length; HV = interval measured during atrial pacing at a cycle length of $600 \mathrm{~ms}$; SCL = sinus cycle length; VERP = ventricular effective refractory period measured at a basic drive train cycle length of $600 \mathrm{~ms}$. 
a clinically apparent sympatholytic effect, no adverse effects related to sympatholysis were noted in the patients in this study treated with amiodarone.

The overall dropout rates for sotalol and amiodarone during the loading period were $19 \%$ and $11 \%$, respectively. In prior studies, the short-term incidence of adverse effects during treatment with sotalol and amiodarone have been reported to range from $13 \%$ to $16 \%, 1,13$ and from $0 \%$ to $5 \%, 6,14$ respectively. Therefore, amiodarone may be better tolerated than sotalol over the short term. However, the long-term organ toxicity of amiodarone may counteract any short-term advantage over sotalol.

Comparison with previous studies: The $24 \%$ efficacy of sotalol in the present study is at the lower end of the $24 \%$ to $67 \%$ range of efficacies reported in prior studies that have used electrophysiologic testing to evaluate sotalol. ${ }^{11,15-20}$ This wide range in the efficacy of sotalol in the treatment of VT may be due to several factors, including heterogeneous patient populations and different techniques for the assessment of drug efficacy. For example, in the Electrophysiology Study Versus Electrocardiographic Monitoring study, ${ }^{1}$ the efficacy of sotalol as judged by electrophysiologic testing was $35 \%$, but in contrast to the present study, not all patients underwent programmed stimulation with triple extrastimuli. Two recent reports have demonstrated that drug efficacy may be overestimated when an incomplete stimulation protocol is used during electropharmacologic testing. ${ }^{21,22}$

The $41 \%$ efficacy of amiodarone in the present study is well within the $29 \%$ to $85 \%$ range of efficacies reported in prior studies. ${ }^{6,23-26}$ Although the loading regimen for amiodarone has varied in these prior studies, a recent study demonstrated that the short-term electrophysiologic effects of amiodarone may be independent of the total dosage of drug received over 10 days as long as a minimal dosage of $1,200 \mathrm{mg} /$ day is given, as was the case in the present study and in most prior studies. ${ }^{6}$

Aside from the present study, the only other randomized comparison of sotalol and amiodarone was a study in which the efficacies of the 2 drugs were assessed in a heterogeneous group of patients solely by clinical followup. ${ }^{27}$ As in the present study, the 2 drugs did not differ significantly in efficacy. Treatment was judged to have been successful in $55 \%$ of patients treated with sotalol compared with $53 \%$ of patients treated with amiodarone.

Study limitations: This study was limited to patients with coronary artery disease and sustained VT with programmed stimulation. Therefore, the results may not necessarily apply to patients with other types of heart disease.

A second potential limitation of this study is the relatively small sample size. Although the success rates of sotalol and amiodarone were not significantly different, there was a trend favoring amiodarone. It is possible that with a larger sample size, the difference in success rates between the 2 drugs would be significant.

A third potential limitation of this study is the dose titration of sotalol to $480 \mathrm{mg} /$ day. Young et $\mathrm{al}^{28}$ demonstrated higher efficacy with $640 \mathrm{mg} /$ day of sotalol. It is possible that with a higher sotalol dosage, there may have been a clinical difference between the 2 drugs.
However, the higher dosage may have resulted in a higher dropout rate due to adverse effects.

Clinical implications: This study has demonstrated that amiodarone and sotalol do not differ significantly in their ability to suppress the induction of sustained monomorphic VT in patients with coronary artery disease. Therefore, in patients who may be appropriate candidates for treatment with a class III antiarrhythmic agent, the decision to use amiodarone or sotalol should be based more on a consideration of the potential adverse effects and toxicities of these 2 drugs, rather than on their relative efficacy. The potential for torsades de pointes and side effects related to $\beta$ blockade with sotalol must be weighed against the potential long-term organ toxicity of amiodarone. For example, in patients who have congestive heart failure, amiodarone may be the more appropriate choice, while in younger patients who do not have severe left ventricular dysfunction, sotalol may be more appropriate.

With the advent of nonthoracotomy lead systems and antitachycardia pacing algorithms, the implantable cardioverter-defibrillator is playing an increasing role in the management of patients with sustained VT. However, insertion of an implantable cardioverter-defibrillator may not always eliminate the need for antiarrhythmic drug therapy. Even when antiarrhythmic drugs are ineffective in suppressing episodes of VT, they may be helpful by slowing the rate of VT (e.g., to facilitate the use of antitachycardia pacing or to decrease the hemodynamic impact of the VT and the probability of syncope). The results of this study suggest that amiodarone may be preferable to sotalol when used in this context because it slows VT approximately twice as much as does sotalol.

1. Mason JW, for The Electrophysiolugy Study Versus Electrocardiographic Monitoring (ESVEM) Investigators. A comparison of seven antiarthythmic drugs in patients with ventricular tachyarrhythmias. $N$ Engl J Med 1993;329:452-458.

2. Kadish AH, Schmaltz S, Morady F. Variability in the measurement of human ventricular refractoriness. PACE 1991;14:1393-1401.

3. Morady F, Kadish A, deBuitleir M, Kou WH, Calkins H, Schmaltz S, Rosenheck S. Sousa J. Prospective comparison of a conventional and an accelerated protocol for programmed ventricular stimulation in patients with coronary artery disease. Circulation 1991;83:764-773.

4. Kadish AH, Buxton AE, Waxman HL, Flores B, Josephson ME, Marchiinski FE Usefulness of electrophysiologic study to determine the clinical tolerance of arrhythmia recurrences during amiodarone therapy. $J$ Am Coll Cardiol 1987;10:90-96.

5. Waller TJ, Kay HR, Spielman SR, Kutalek SP, Greenspan AM. Reduction in sudden death and total mortality by antiarhythmic therapy evaluated by electrophysiologic drug testing: criteria of efficacy in patients with sustained ventricular tachyarrhythmia. J Am Coll Cardiol 1987;10:83-89.

6. Kalbfleisch SJ, Williamson B, Man KC, Vorperian V, Hummel JD, Hasse C, Strickberger SA, Calkins H, Langberg JJ, Morady F. Prospective, randomized comparison of conventional and high dose loading regimens of amiodarone in the treatment of ventricular tachycardia. J Am Coll Cardiol 1993:22:1723-1729.

7. Zhu J, Haines DE, Lerman BB, DiMarco JP. Predictors of efficacy of amiodarone and characteristics of recurrence of arrhythmia in patients with sustained ventricular tachycardia and coronary artery disease. Circulation 1987;76:802-809. 8. Horowitz LN, Greenspan AM, Spielman SR, Webb CR, Morganroth J, Rotmensch H, Sokoloff NM, Rae AP, Segal BL, Kay HR. Usefulness of electrophysiologic testing in evaluation of amiodarone therapy for sustained ventricular tachyarrhythmias associated with coronary heart disease. AmJ Cardiol 1985;55:367-371. 9. Yazaki Y, Haffajee CZ, Gold RL, Bishop RL, Alpert JS. Electrophysiologic predictors of long-term clinical outcome with amiodarone for refractory ventricular tachycardia secondary to coronary artery disease. Am J Cardial 1987;60:293-297. 10. Nademanee K, Feld G, Hendrickson J, Singh PN, Singh BN, Electrophysiologic and antiarrhythmic effects of sotalol in patients with life-threatening ventricular tachyarrhythmias. Circulation 1985;72:555-564.

11. Kuchar DL, Garan H, Venditti FJ, Finkelstein D, Rottman JN, McComb J, McGovem BA, Ruskin JN. Usefulness of sotalol in suppressing ventricular tachycardia or ventricular fibrillation in patients with healed myocardial infarcts. Am J 
Cardiol 1989;64:33-36.

12. Morady F, DiCarlo L, Krol RB, Baerman JM, deBuitleir M. Acute and chronic effects of amiodarone on ventricular refractoriness, intraventricular conduction and ventricular tachycardia induction. J Am Coll Cardiol 1986;7:148-157.

13. Kehoe RF, Zheutlin TA, Dunnington CS, Mattioni TA, Yu G, Spangenberg RB. Safety and efficacy of sotalol in patients with drug-refractory sustained ventricular tachyarthythmias. Am J Cardiol 1990;65:58A-64A.

14. Raeder EA, Podrid PJ, Lown B. Side effects and complications of amiodarone therapy. Am Heart J 1985; 109:975-983.

15. Gonzalez R, Scheinman MM, Herre JM, Griffin JC, Sauve MJ, Sharkey H. Usefuiness of sotalol for drug-refractory malignant ventricular arrhythmias. $J \mathrm{Am}$ Coll Cardiol 1988;12:1568-1572

16. Ruder MA. Ellis T, Lebsack C, Mead RH, Smith NA, Winkle RA. Clinical experience with sotalol in patients with drug-refractory ventricular arrhythmias. $J$ Am Coll Cardiol 1989;13:145-152.

17. Singh SN, Cohen A, Chen Y, Wish M, Thoben-O'Grady L, Peralba J, Gottdiener J, Fletcher RD. Sotalol for refractory sustained ventricular tachycardia and nonfatal cardiac arrest. Am $J$ Cardiol 1988;62:399-402.

18. Senges J, Lengfelder W, Jauernig R, Czygan E, Brachmann J, Rizos I, Cobbe $\mathrm{S}, \mathrm{Kubler} \mathrm{W}$. Electrophysiologic testing in assessment of therapy with sotalol for sustained ventricular tachycardia. Circulation 1984;69:577-584.

19. Steinbeck G, Bach $P$, Haberl R. Electrophysiologic and antiarrhythmic efficacy of oral sotalol for sustained ventricular tachyarrhythmias: evaluation by programmed stimulation and ambulatory electrocardiogram. $J$ Am Coll Cardiol 1986;8:949-958. 20. Nademanee K, Singh BN. Effects of sotalol on ventricular tachycardia and fibrillation produced hy programmed electrical stimulation: comparison with other antiarrhythmic agents. Am J Cardiol 1990;65:53A-57A

21. Winters SL, Rubenstein DG, Ip J, Goldman DS, Banas JS, Patel PV, Gomes A. Truncated serial drug stimulation protocols may limit the interpretation of ESVEM (abstr). I Am Coll Cardiol 1993;21:328A.

22. Mann DE, Kelly PA, Fuenzalida CE, Bailey WM, Reiter MJ. Influence of a third extrastimulus in defining effective drug therapy during serial electrophysiologic testing (abstr). $J$ Am Coll Cardiol 1993;21:328A

23. Heger JJ, Prystowsky EN, Jackman WM, Naccarelli GV, Warfel KA, Rinkenberger RL, Zipes DP. Clinical efficacy and electrophysiology during long-term therapy for recurrent ventricular tachycardia or ventricular fibrillation. $N$ Engl J Med 1981;305:539--545.

24. Fogoros RN, Anderson KP, Winkle RA, Swerdlow CD, Mason JW. Amiodarone: clinical efficacy and toxicity in 96 patients with recurrent, drug-refractory arrhythmias. Circulation 1983;68:88-94.

25. Nademanee K, Singh BN, Canrom DS, Weiss J, Feld G, Stevenson WG. Control of sudden recurrent arrhythmic deaths: role of amiodarone. Am Heart J 1983; 106:895-901.

26. McGovern B, Garan $H$, Malacoff RF, DiMarco JP, Grant G, Sellers TD, Ruskin JN. Long-term clinical outcome of ventricular tachycardia or fibrillation treated with amiodarone. Am J Cardiol 1984;53:1558-1563.

27. Amiodarone vs Sotalol Study Group. Multicentre randomized trial of sotalol vs amiodarone for chronic malignant ventricular tachyarrhythmias. Eur Heart $J$ 1989;10:685-694.

28. Young GD, Kerr CR, Mohama R, Boone J, Yeung-Lai-Wah JA. Efficacy of sotalol guided by programmed electrical stimulation for sustained ventricular arthythmias secondary to coronary artery disease. Am I Cardiol 1994;73:677-682. 